\title{
Quantification of irradiation defects in beta-silicon carbide using Raman spectroscopy ${ }^{\text {a) }}$
}

\author{
T. Koyanagi, ${ }^{1, b)}$ M. J. Lance, ${ }^{1}$ and Y. Katoh, ${ }^{1}$ \\ ${ }^{1}$ Oak Ridge National Laboratory, Oak Ridge, Tennessee 37831, USA
}

Raman spectra from polycrystalline beta-silicon carbide $(\mathrm{SiC})$ were collected following neutron irradiation at $380-1180^{\circ} \mathrm{C}$ to 0.011-1.87 displacement per atom. The longitudinal optical (LO) peak shifted to a lower frequency and broadened as a result of the irradiation. The changes observed in the LO phonon line shape and position in neutron-irradiated SiC are explained by a combination of changes in the lattice constant and Young's modulus, and the phonon confinement effect. The phonon confinement model reasonably estimates the defect-defect distance in the irradiated $\mathrm{SiC}$, which is consistent with results from previous experimental studies and simulations.

\section{INTRODUCTION}

Silicon carbide $(\mathrm{SiC})$ has been extensively investigated as a high temperature material for use in nuclear core structures $^{1}$. Core materials are exposed to extreme environments, including high temperatures and neutrons during operation, which results in significant changes in the thermo-physical and mechanical properties ${ }^{1}$. The changes are due to irradiationinduced defects including Frenkel pairs, antisite defects, and clusters of interstitials and vacancies. Transmission electron microscopy (TEM) has been systematically applied to understand radiation temperature and neutron dose dependencies on the microstructural evolution of $\mathrm{SiC}^{2}$. However, the correlation between microstructures and macroscopic properties, such as volumetric swelling and irradiation creep, is still poorly understood, as TEM-visible defects account for only a small fraction of the changes in those properties ${ }^{3}$. This suggests the importance of evaluating atomistic defects, that cannot be observed by conventional TEM techniques because of the resolution limit (typically $\sim 1 \mathrm{~nm}$ in TEM dark field imaging). Atomistic defects in irradiated $\mathrm{SiC}$ have been successfully evaluated in detail using various techniques, including positron annihilation spectroscopy ${ }^{4}$, electron paramagnetic resonance ${ }^{5}$, photoluminescence $^{6}$, deep-level transient spectroscopy ${ }^{7}$ and Raman spectroscopy ${ }^{8,9,10,11}$. We propose a new approach to characterize irradiation defects in SiC using Raman spectroscopy to provide a better understanding of irradiation effects on the microstructure of SiC.

\footnotetext{
a) Notice: This manuscript has been authored by UT-Battelle, LLC under Contract No. DE-AC05-00OR22725 with the U.S. Department of Energy. The United States Government retains and the publisher, by accepting the article for publication, acknowledges that the United States Government retains a non-exclusive, paid-up, irrevocable, world-wide license to publish or reproduce the published form of this manuscript, or allow others to do so, for United States Government purposes. The Department of Energy will provide public access to these results of federally sponsored research in accordance with the DOE Public Access Plan (http://energy.gov/downloads/doe-public-access-plan).

b) Corresponding author. Electronic mail: koyanagit@ornl.gov
} 
Tiong et al. ${ }^{12}$ proposed an approach to estimate the sizes of undamaged regions in irradiated semiconductors using Raman spectroscopy. In that study, the analysis interpreted the Raman spectra of ion-irradiated GaAs using the phonon

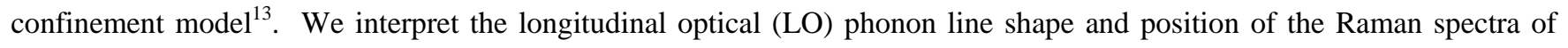
neutron-irradiated $\mathrm{SiC}$ using the phonon confinement model to quantify the radiation damage. This analysis considers the irradiation-induced changes in the modulus and lattice constant and the phonon conferment effects. The approach to analyzing the LO phonon line in this study is different from the approach used in previous sutudies ${ }^{8,9,10,11}$. The interests in the previous Raman spectroscopy studies, such as radiation-induced amorphization and analysis of $\mathrm{Si}-\mathrm{Si}$ and $\mathrm{C}-\mathrm{C}$ homonuclear bands, are beyond the focus of this study.

\section{EXPERIMENTAL METHODS}

Polycrystalline, chemical vapor deposited (CVD) $\beta$-SiC (high resistivity grade, Dow Chemical Co., Marlborough, Massachusetts) was used in this investigation. The material purity was $\geq 99.9995 \%$. The unirradiated material contained preexisting defects such as grain boundaries, twin boundaries, and stacking faults. The average coherent grain size of $\sim 200$ $\mathrm{nm}$ was confirmed by X-ray diffraction (XRD) and TEM, and nearly isotropic grain growth was observed ${ }^{14}$. Neutron irradiations were conducted in the High Flux Isotope Reactor at Oak Ridge National Laboratory. The irradiation temperature and neutron fluence ranged from 380 to $1180^{\circ} \mathrm{C}$ and 0.011 to $1.87 \times 10^{25} \mathrm{n} / \mathrm{m}^{2}$ [E $\left.>0.1 \mathrm{MeV}\right]$, respectively. These fluences correspond with nominal doses of 0.01 to 1.87 displacement per atom (dpa) in SiC. The irradiation conditions are summarized in TABLE I. The irradiation temperatures were determined by post-irradiation measurement of the dimensional changes of the $\mathrm{SiC}$ specimens upon annealing, using a dilatometer ${ }^{15}$.

Raman spectra of the SiC specimens were recorded at room temperature with a DilorXY800 (Horiba Scientific, Edison, NJ) Raman microprobe using an Innova 308C $\mathrm{Ar}^{+}$ion laser (Coherent Laser Group, Santa Clara, CA) at $515 \mathrm{~nm}$ and $50 \mathrm{~mW}$ output power. The laser was focused using a 50x objective to a spot roughly $2 \mu \mathrm{m}$ in diameter. Calibration of the spectra was conducted using a peak from the argon laser at $116.2 \mathrm{~cm}^{-1}$. Five spectra were collected and averaged for each specimen, which enabled us to evaluate $\sim 500$ coherent grains. Since the pre-existing defects were not changed by irradiation ${ }^{3}$ and the defect density was much smaller than the irradiation-defect density, the preexisting defects could be ignored during the analysis of the Raman spectra.

Other physical property and microstructural observations were also conducted to aid in analysis. The volumetric swelling of the specimens was calculated based on the length change of the specimen measured using a conventional micrometer, assuming isotropic swelling in all directions. The original specimen length was $\sim 40 \mathrm{~mm}$, and the accuracy of the evaluation of the volumetric swelling was $\pm 0.08 \%$. The microstructure of a selected specimen was examined by TEM. The TEM foil 
was prepared using a focused ion beam (FEI, Versa 3D DualBeam) operated at 16 and $30 \mathrm{kV}$ for rough milling and 2 and 5 $\mathrm{kV}$ for final thinning. TEM observation was conducted using a JEOL JEM2100F operated at $200 \mathrm{kV}$. The lattice constants were obtained using high-energy XRD in another work ${ }^{16}$.

TABLE I. Summary of irradiation conditions and properties of SiC materials. Parentheses indicate the standard deviation. The error in the determination of the lattice constant and volumetric swelling was less than $0.00008 \AA$ and $\pm 0.08 \%$, respectively.

\begin{tabular}{ccccc}
\hline \hline $\begin{array}{c}\text { Irradiation } \\
\text { temperature } \\
{\left[{ }^{\circ} \mathrm{C}\right]}\end{array}$ & $\begin{array}{c}\text { Neutron } \\
\text { dose }\end{array}$ & $\begin{array}{c}\text { Volumetric } \\
\text { swelling } \\
{[\%]}\end{array}$ & $\begin{array}{c}\text { Lattice } \\
\text { constant } \\
{[\AA]^{16}}\end{array}$ & $\begin{array}{c}\text { Estimated } \\
\text { correlation } \\
\text { length [nm] }\end{array}$ \\
\hline $\begin{array}{c}\text { Uniradiated } \\
380\end{array}$ & 0 & 0 & 4.36075 & Not applicable \\
380 & $0.011 \mathrm{dpa}$ & 0.48 & 4.36481 & $2.7(0.5)$ \\
540 & $0.11 \mathrm{dpa}$ & 1.50 & 4.38131 & $0.6(0.0)$ \\
540 & $0.11 \mathrm{dpa}$ & 0.39 & 4.36302 & $2.0(0.3)$ \\
790 & $0.11 \mathrm{dpa}$ & 0.34 & 4.36650 & $0.8(0.0)$ \\
760 & $1.4 \mathrm{dpa}$ & 0.76 & 4.36800 & $0.8(0.5)$ \\
1180 & $1.9 \mathrm{dpa}$ & 0.28 & 4.36221 & $15(5)$ \\
\hline \hline
\end{tabular}

\section{RESULTS AND DISCUSSION}

\section{A. ANALYSIS OF RAMAN SPECTRA}

The recorded Raman spectra of the unirradiated and irradiated specimens are shown in FIG. 1. The peaks of the transverse optical (TO) and LO phonon lines of $\beta$-SiC appear at around 800 and $950 \mathrm{~cm}^{-1}$, respectively. Additional broad bands at $\sim 200, \sim 520, \sim 1400$ and $\sim 1600 \mathrm{~cm}^{-1}$ were observed only in irradiated specimens. Those peaks are considered to be related to vibrations of $\mathrm{Si}-\mathrm{Si}\left(\sim 200\right.$ and $\left.\sim 520 \mathrm{~cm}^{-1}\right)$ and $\mathrm{C}-\mathrm{C}\left(\sim 1400\right.$ and $\left.\sim 1600 \mathrm{~cm}^{-1}\right)$ bonds ${ }^{9}$. In all cases, the peak intensity of the TO and LO phonon lines decreased following irradiation. In addition, there was a significant peak shift to lower frequencies and broadening of the TO and LO phonon peaks, depending on the irradiation conditions. These qualitative changes in the Raman spectra were also reported in previous work ${ }^{8,9,10,11}$. Several factors can contribute to changes in the Raman peak position and/or linewidth of the TO and LO peaks. These include changes in the lattice constant and phonon confinement caused by the reduction of undisturbed regions due to defects.

Irradiation defects in $\mathrm{SiC}$ are known to cause lattice expansion (elastic strain) ${ }^{2}$. The effect of elastic strain on phonon Raman bands of $\beta$-SiC were studied by Olego et al. ${ }^{17}$, who found a linear correlation between Raman peak positions and elastic strain. For irradiated SiC, the peak shift resulting from elastic strain will change as the crystallite size decreases 
because more defects will increase the strain-induced peak shift, which is related to the anharmonicity of the atomic bonding. Since the lattice expansion of the irradiated $\mathrm{SiC}$ was almost isotropic, we assume that the change in lattice spacing caused by applied stress and irradiation defects had the same effect on the peak shift. This assumption is reasonable based on the comparison of calculated and experimental TO lines shown below. The strain dependence of frequency $(\omega)$ of the TO and LO mode against the relative lattice constant can be given (in units of $\mathrm{cm}^{-1}$ ) by ${ }^{17}$

$$
\omega(T O)=797-3734 \frac{E_{\text {unir }}}{E_{\text {irr }}} \frac{\left(a_{\text {irr }}-a_{\text {unir }}\right)}{a_{\text {unir }}}, \quad \omega(L O)=973-4532 \frac{E_{\text {unir }}}{E_{\text {irr }}} \frac{\left(a_{\text {irr }}-a_{\text {unir }}\right)}{a_{\text {unir }}}
$$

where $E$ and $a$ are the Young's modulus and lattice constant, respectively. The subscripts unir and irr indicate properties of unirradiated and irradiated materials, respectively. The lattice constant was obtained from XRD ${ }^{16}$ (Table I). The change in modulus by radiation is given $b^{2}$ :

$$
E_{\text {irr }}=E_{\text {unir }}(1-6.974 S) \quad\left(T_{\text {irr }}<\sim 1000^{\circ} \mathrm{C}\right)
$$

where $S$ is volumetric swelling and $T_{i r r}$ is the irradiation temperature. As mentioned previously, the swelling was determined from the length change of the specimen (Table I). The reduction in the Young's modulus was up to $10 \%$ in this study, and the change in Young's modulus was negligible beyond the irradiation temperature of $1000^{\circ} \mathrm{C}^{2}$. Using Equations $(1)$ and $(2)$, we calculated and then compared the peak position resulting from the change in the lattice constant with the observed peak shifts in FIG. 2. The calculated change in the lattice constant contributed to peak shifts of the TO and LO lines of up to 21 and $25 \mathrm{~cm}^{-1}$, respectively. The peak shift of the TO phonon lines due to irradiation defects is fully explained by Equation (1). However, Eq. (1) does not explain the peak shift of the LO lines. The disagreement between the experimental and calculated shift becomes more significant for a larger lattice expansion. The remainder of the LO peak shift can be explained by phonon confinement effects due to irradiation defects, as will be shown later. The phonon confinement effect on the TO line is expected to be minimal owing to the phonon dispersion relation of $\beta-\mathrm{SiC}^{18}$.

The downshifting and asymmetric broadening of the Raman lines due to the nanostructures of the materials has been reported for various semiconductors ${ }^{19}$. Richter et al. ${ }^{13}$ reported a quantitative explanation of the Raman spectra of microcrystalline silicon using a phonon-confinement model, which was also applied to ion-irradiated $\mathrm{GaAs}^{12}$ and $\mathrm{MoS}_{2}{ }^{20}$ materials. In an infinite crystal, only phonons from the center of the Brillouin zone $(\mathrm{q} \sim 0)$ are involved in first-order Raman scattering because of wave vector selection rules; but in a finite crystal, phonons can be confined in space by grain boundaries or defects. This results in uncertainty in the phonon momentum, and allows for phonons with $\mathrm{q} \neq 0$ to contribute to the Raman spectrum. In the phonon confinement model the contribution of modes to the Raman spectrum is calculated by assuming a Gaussian distribution function of phonon states in $q$ space. The $q$-dependent weight factor $C(q)$ can be written as $^{13}$ : 


$$
|C(q)|^{2}=\exp \left(-\frac{q^{2} L^{2}}{4}\right)
$$

where $q$ is expressed in units of $2 \pi / a$, where $a$ is the lattice constant, and $L$ is the diameter of the correlation region. This weight factor was used for an ion-irradiated material ${ }^{12,20}$ and recognized as an appropriate value ${ }^{21}$. Assuming uniform distribution of a spherical undamaged region in the irradiated material, the first-order Raman spectrum at frequency $w, I(w)$, can be written as integrating these contributions over the complete Brillouin zone ${ }^{13}$ :

$$
\mathrm{I}(\mathrm{w})=\int \frac{|C(q)|^{2} d^{3} q}{[w-w(q)]^{2}+\left(\frac{\Gamma_{0}}{2}\right)^{2}}
$$

where $w(q)$ is the phonon dispersion curve and $\Gamma_{0}$ is the natural linewidth of the zone-center optical phonon in the bulk $(\sim 3.0$ $\mathrm{cm}^{-1}$ in this study). The dispersion $w(q)$ of the LO phonon in $\beta$-SiC is approximated as ${ }^{12}: w(q) \sim A+B \cos (\pi q)$, where $A=$ $903 \mathrm{~cm}^{-1}$ and $B=70 \mathrm{~cm}^{-1}$. This equation is shown to reasonably describe the dispersion of the LO phonon in $\beta-\mathrm{SiC}$ along the $<110>$ direction $^{18}$.

FIGURE 3(a) shows calculated Raman spectra of confined LO phonon lines in $\beta$-SiC based on Eq. (4). Both the asymmetric broadening of the line shape and the shift of the peak position toward the lower-frequency side became significant when the diameter of the correlation region was less than $\sim 5 \mathrm{~nm}$. This means that the phonon confinement model enabled an accurate evaluation of the size of the undistorted area only for highly damaged SiC. We will show later that the correlation length was less than $5 \mathrm{~nm}$ in most cases of this study, so the use of the phonon confinement model is an appropriate approach to quantifying the radiation damage. The Raman spectra of irradiated $\mathrm{SiC}$ can be calculated by the summation of Eqs. (1) and (4), which is shown for the experimental Raman spectra for low-dose (FIG. 3 (b)) and high-dose (FIG. 3 (c)) irradiations. The Gaussian peaks used for fitting are known as the Raman bands of disordered or amorphous SiC with peak positions of $\sim 940 \mathrm{~cm}^{-1}$ for the low-dose case and $\sim 870 \mathrm{~cm}^{-1}$ for the high dose case ${ }^{9}$. The Gaussian peak position tends to decrease as the lattice expansion increases, which might be useful as an indication of radiation damage. The experimental LO phonon line was fitted with the calculated LO line based on the peak position. For of the material irradiated at a low-dose, the fitting was straightforward because of the relatively sharp line shape. In the case of high-dose irradiation, there may have been certain variations in the fitting due to the broad LO line. However, the variation of the correlation length was small (typically less than $0.5 \mathrm{~nm}$ ) because the calculated line shape and position were highly sensitive to small changes in the correlation lengths. The combined effects of the phonon confinement and change in the lattice constant explain the experimentally observed LO peak shifts up to $49 \mathrm{~cm}^{-1}$ and the LO peak broadening for all cases. The estimated correlation lengths are summarized in Table 1 . Except for the case of irradiation at $1180^{\circ} \mathrm{C}$, the correlation lengths were less than $4 \mathrm{~nm}$. 


\section{B. VALIDATION OF ANALYSIS OF THE RAMAN SPECTRA}

Validation of the analysis of the Raman spectra was carried out using TEM. A weak beam dark field micrograph (the inserted image in FIG. 4) shows high-density defects in $\mathrm{SiC}$ irradiated at $380{ }^{\circ} \mathrm{C}$ to $0.11 \mathrm{dpa}$, which are known to be interstitial clusters with an unidentified habit plane and Burgers vector due to the small size ${ }^{3}$. The average defect diameter and the density were $\sim 1.5 \mathrm{~nm}$ and $\sim 1 \times 10^{24} \mathrm{~m}^{-3}$, respectively. The average distance between the defects, i.e., average radius from a defect to the surrounding defects, was estimated to be $\sim 7 \mathrm{~nm}$, assuming the defect size was much smaller than the defect distance. This estimated defect distance was one order of magnitude larger than the value obtained from the Raman spectra $(0.6 \mathrm{~nm})$ if it is assumed that the correlation length in the phonon confinement model corresponds to the average defect distance. This is likely because TME could not capture the point defects.

FIGURE 4 shows the defect distances in irradiated SiC evaluated by different methods. The definition of defect distance for Raman is the correlation length, and the spacing between defects is used as the TEM defect distance. Additional TEM results from Katoh et al. ${ }^{22}$ are also included for comparison. A third experimental approach to evaluating the defect density is to analyze temperature-dependent thermal conductivity ${ }^{23,24}$. This method is sensitive to any defects causing phonon scattering. The defect mean free path, from the thermal conductivity results, is used as the average defect distance in FIG. 4. Crocombettea et al. ${ }^{25}$ conducted a simulation study of the thermal resistance of point defects and estimated the vacancy concentration in irradiated SiC. Note that these previous studies also investigated neutron-irradiated, high-purity, polycrystalline CVD $\beta$-SiC. Defect distances were observed that are similar to the results from this study and the historical thermal conductivity and simulation studies. The simulation work did not consider interstitial defects, but the agreement between this work and the simulations indicates that the phonon analysis of Raman spectra can capture significant numbers of the atomistic defects that cannot be observed in TEM. A similar defect distance was also found from the Raman spectra, and the thermal conductivity analysis provided additional validation of this method of Raman spectra analysis. The increasing defect distance with higher irradiation temperature can be explained by defect recovery processes, i.e., the annihilation of Frenkel pairs, which was enhanced with increasing temperature ${ }^{2}$. The significant increase in the defect distance from $\sim 800$ to $1180{ }^{\circ} \mathrm{C}$ is attributed to the vacancy mobility above $\sim 1000{ }^{\circ} \mathrm{C}$ in $\mathrm{SiC}^{2}$. In addition, the defect distance tended to decrease with increasing swelling and neutron dose (up to $1.4 \mathrm{dpa}$ ) (TABLE I), which is quite reasonable. In summary, the correlation length obtained from Raman spectroscopy is consistent with the previous non-TEM experimental and simulation studies. Although Fano interference as a result of electron-phonon interactions can cause a low-lying frequency mode in Raman spectra, as observed in silicon ${ }^{26}$, this phenomenon was not observed in the LO phonon line of 
$\mathrm{SiC}^{27}$. LO-phonon-plasmon coupling (LOPC) is another possible cause of a peak shift to a lower frequency and peak asymmetry. However, the LOPC mode caused peak asymmetry only at higher frequencies in both n- and p-type beta $\mathrm{SiC}^{28,}{ }^{29}$, which is not consistent with our observations. In addition, transmutation elements such as $\mathrm{P}, \mathrm{Mg}$, and Be produced during neutron irradiation are believed to have an ignorable effect on Raman spectra because of their limited amounts ${ }^{30}$. Moreover, a high-density stacking fault is another possibility as a cause the asymmetric LO peak broadening in beta $\mathrm{SiC}^{31}$. However, this type of defect was not dominant in the irradiated $\mathrm{SiC}^{2,3}$. Therefore, the irradiation effect on the $\mathrm{LO}$ line is explained by the phonon confinement effect and lattice strain.

\section{CONCLUSIONS}

This study proposes and provides preliminary validation of an experimental method to evaluate and quantify defects invisible to conventional TEM. The shift and peak broadening of the LO phonon line in the Raman spectra of SiC resulting from neutron irradiation, is interpreted as a combination of lattice strain and phonon confinement. The phonon confinement model enabled an estimation of the size of the undistorted area, i.e., the defect distance in highly disordered SiC due to the sensitivity to atomic-scale correlation lengths. The estimated defect distances were $<3 \mathrm{~nm}$ and $\sim 15 \mathrm{~nm}$ following irradiation at $380-790{ }^{\circ} \mathrm{C}$ and $1180{ }^{\circ} \mathrm{C}$, respectively. The results are consistent with the results of previous experimental and simulation studies.

\section{ACKNOWLEDGEMENTS}

This work is supported by the U.S. Department of Energy (DOE), Office of Fusion Energy Sciences and Office of Nuclear Energy for the Fuel Cycle Research \& Development program under contact DE-AC05-00OR22725 with Oak Ridge National Laboratory managed by UT-Battelle, LLC. The research is also supported in part by the High Flux Isotope Reactor, which is sponsored by the DOE Office of Basic Energy Sciences. The authors are grateful to A. A. Campbell and J. Nanda (ORNL) for their valuable comments. The XRD experiment shown in this paper was carried out at the National Synchrotron Light Source-II at Brookhaven National Laboratory (BNL), which is supported by the U.S. DOE. The authors would like to thank D. Sprouster and L. Ecker (BNL) for their invaluable efforts and time given to the XRD experiment and the analysis. 


\section{REFERENCES}

${ }^{1}$ Y. Katoh, L.L. Snead, C.H. Henager Jr., T. Nozawa, T. Hinoki, A. Ivekovic, S. Novak, S.M. Gonzalez de Vicente, J. Nucl. Mater 455, 387 (2014).

${ }^{2}$ L.L. Snead, T. Nozawa, Y. Katoh, T.-S. Byun, S. Kondo, and D.A. Petti, J. Nucl. Mater. 371, 329 (2007).

${ }^{3}$ Y. Katoh, S. Kondo, L.L. Snead, J. Nucl. Mater. 382, 170 (2008).

${ }^{4}$ J. Wiktor, X. Kerbiriou, G. Jomard, S. Esnouf, M.-F. Barthe, and M. Bertolus, Phys. Rev. B 89, 155203 (2014).

${ }^{5}$ S. B. Orlinski, J. Schmidt, E. N. Mokhov and P. G. Baranov, Phys. Rev. B 67, 125207 (2003).

${ }^{6}$ J.W. Steeds, Phys. Rev. B 80, 245202 (2009).

${ }^{7}$ M.O. Aboelfotoh, J.P. Doyle, Phys. Rev. B 59, 10823 (1999).

${ }^{8}$ P. Krautwasser, G.M. Begun, and P. Angelini, J Am. Ceram. Soc. 66, 424 (1983).

${ }^{9}$ S Sorieul, J-M Costantini, L Gosmain, L Thome and J-J Grob, J. Phys.: Condens. Matter 18, 5235 (2006).

${ }^{10}$ S. Miro, J.-M. Costantini, S. Sorieul, L. Gosmain and L. Thomé, Phil. Mag. Lett. 92, 633 (2012).

${ }^{11}$ P.F. Wang, Y.F. Ruan, L. Huang, and W. Zhu, J. Appl. Phys. 111, 063517 (2012).

${ }^{12}$ K.K. Tiong, P.M. Amirtharaj, F.H. Pollak, and D.E. Aspnes, Appl. Phys. Lett. 44, 122 (1984).

${ }^{13}$ H. Richter, Z.P. Wang, and L. Ley, Solid State Commun. 39, 625 (1981).

${ }^{14}$ Y. Katoh, L.L. Snead, C.M. Parish, T. Hinoki, J. Nucl. Mater 434, 141 (2013).

15 A.A. Campbell, W.D. Porter, Y. Katoh, L.L. Snead, Nucl. Instrum. Meth. B 370, 370 (2016).

${ }^{16}$ D.J. Sprouster, S.K. Ghose, E. Dooryhee, L.E. Ecker, T. Koyanagi, Y. Katoh, To be submitted.

${ }^{17}$ D. Olego and M. Cardona, Phys. Rev. B 25, 1151 (1982).

${ }^{18}$ J. Serrano, J. Strempfer, M. Cardona, M. Schwoerer-Böhning, H. Requardt, M. Lorenzen, B. Stojetz, P. Pavone, and W.J. Choyke, Appl. Phys. Lett. 80, 4360 (2002).

${ }^{19}$ A.K. Arora, M. Rajalakshmi, T.R. Ravindran and V. Sivasubramanian, J. Raman Spectrosc. 38, 604 (2007).

${ }^{20}$ S. Mignuzzi, A.J. Pollard, N. Bonini, B. Brennan, I.S. Gilmore, M.A. Pimenta, D. Richards, and D. Roy, Phys. Rev. B 91, $195411(2015)$.

${ }^{21}$ I.F. Crowe, M.P. Halsall, O. Hulko, A.P. Knights, R.M. Gwilliam, M. Wojdak, and A.J. Kenyon J. Appl. Phys. 109, 083534 (2011).

${ }^{22}$ Y. Katoh, N. Hashimoto, S. Kondo, L.L. Snead, and A. Kohyama, J. Nucl. Mater 351, 228 (2006).

23 D.J. Senor, G.E. Youngblood, L.R. Greenwood, D.V. Archer, D.L. Alexander, M.C. Chen and G.A. Newsome, J. Nucl. Mater. 317, 145 (2003).

${ }^{24}$ L.L. Snead, S.J. Zinkle, and D.P. White, J. Nucl. Mater 340, 187 (2005).

${ }^{25}$ J.-P. Crocombettea and L. Proville, Appl. Phys. Lett. 98, 191905 (2011).

${ }^{26}$ R. Gupta, Q. Xiong, C.K. Adu, U.J. Kim, and P.C. Eklund, Nano Lett., 3, 627 (2003).

${ }^{27}$ T. Mitani, S. Nakashima, K. Kojima, T. Kato,and H. Okumura, J. Appl. Phys. 112, 043514 (2012).

${ }^{28}$ H. Yugami, S. Nakashima, A. Mitsuishi, A. Uemoto, M. Shigeta, K. Furukawa, A. Suzuki, and S. Nakajima, J. Appl. Phys. 61, 354 (1987).

${ }^{29}$ Z. Li, W. Zhou, X. Su, Y. Huang, G. Li, and Y. Wang, J. Am. Ceram. Soc., 92, 2116 (2009).

${ }^{30}$ H.L. Heinisch, L.R. Greenwood, W.J. Weber, R.E. Williford, J. Nucl. Mater 327, 175 (2004).

${ }^{31}$ S. Rohmfeld, M. Hundhausen, and L. Ley, Phys. Rev. B 58, 9858 (1998). 
FIG. 1 Raman spectra of unirradiated and irradiated SiC. Dotted lines indicate the peak positions of TO or LO phonon lines of the unirradiated material.

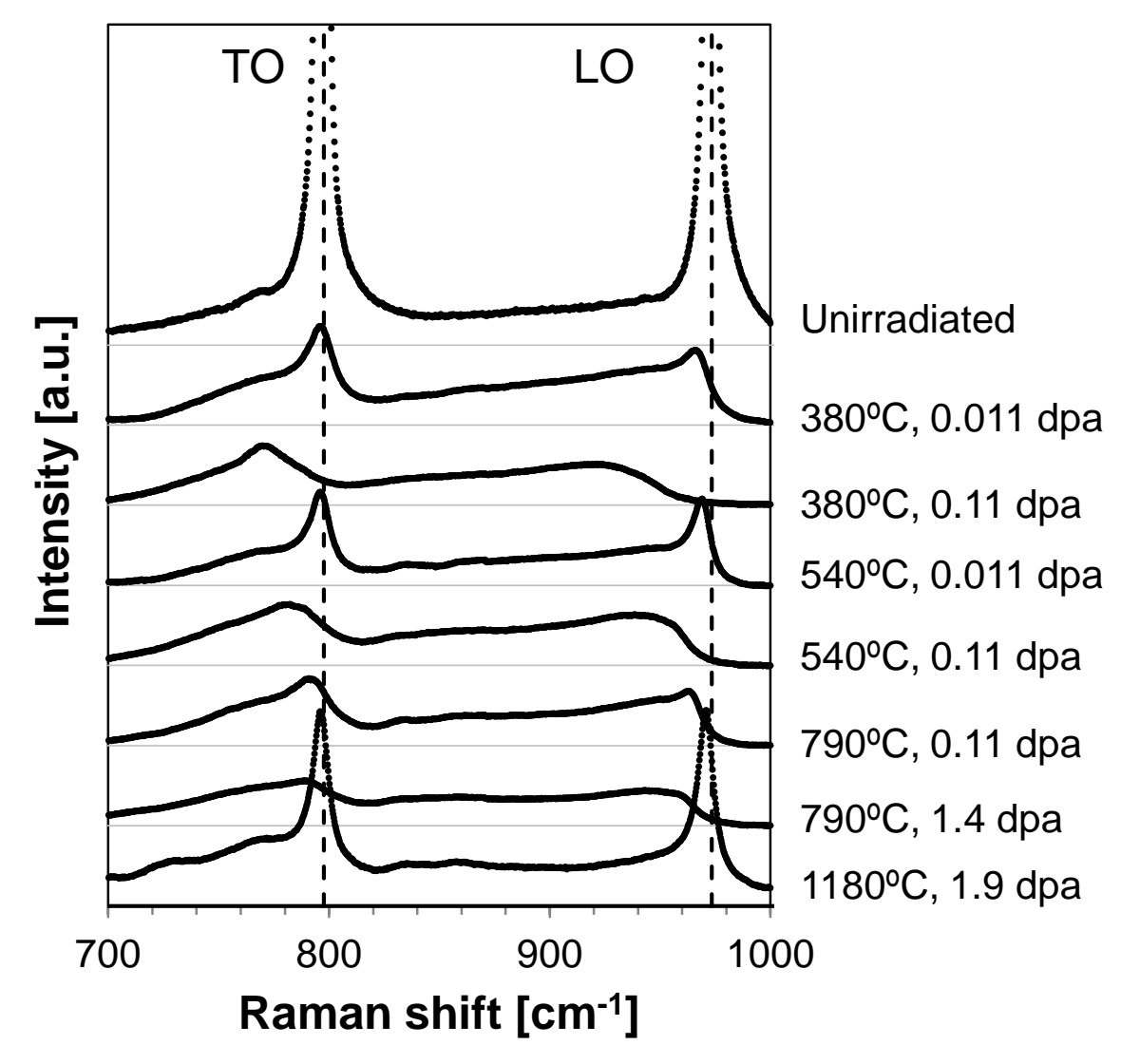


FIG. 2 Comparison of measured and calculated peak positions of SiC TO and LO phonon lines. The calculated values are based on results from equations (1).

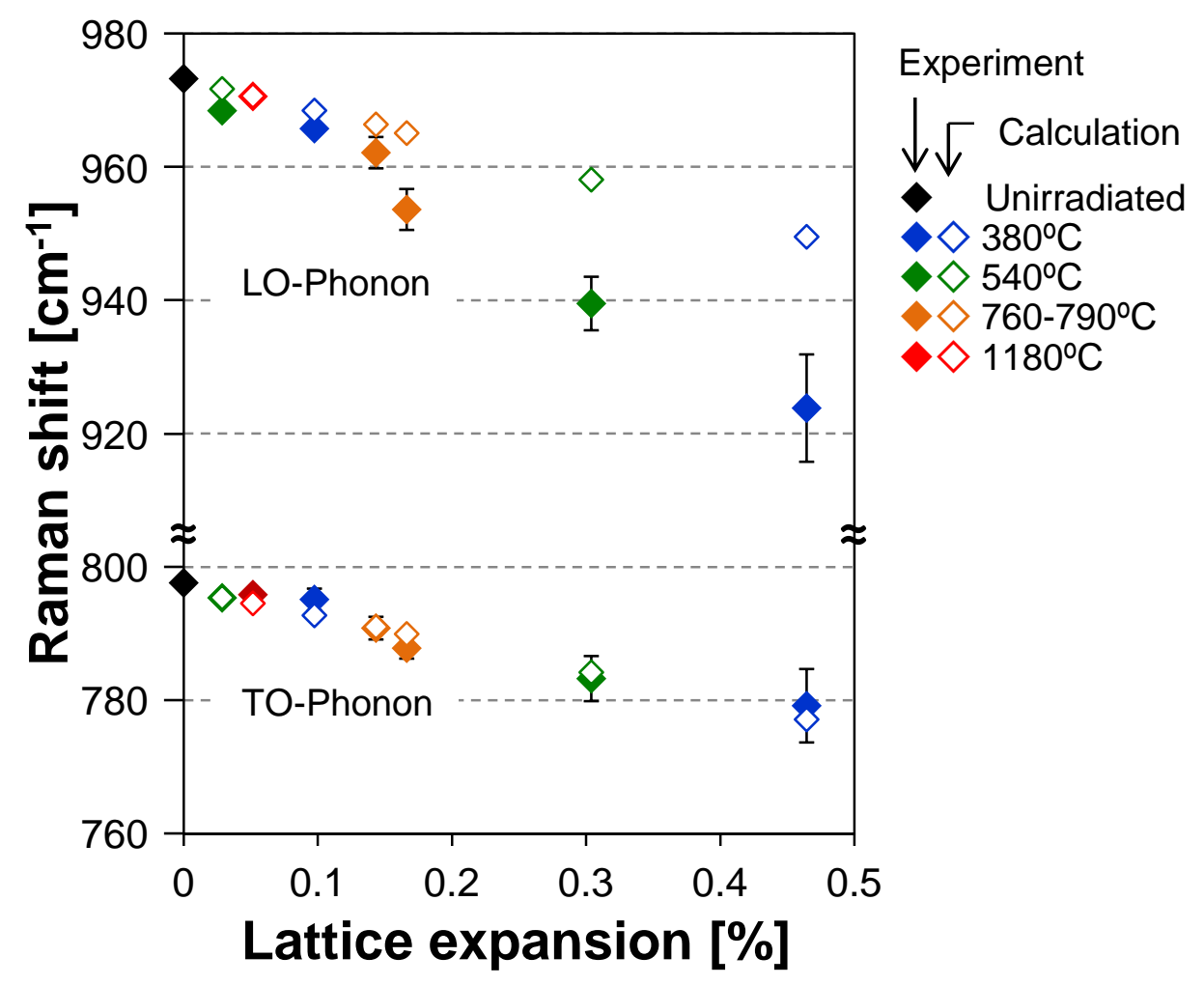


FIG. 3 (a) Calculated Raman spectra of confined LO phonon lines of $\beta$-SiC with various size of correlation diameters. Raman spectra of irradiated $\mathrm{SiC}$ at $540^{\circ} \mathrm{C}$ to (b) $0.011 \mathrm{dpa}$ and (c) 0.11 dpa are compared with the calculated spectra considering changes in lattice constant and the phonon confinement effect. The experimental spectra are in good agreement with the sum of the calculated spectra and a Gaussian peak known as the Raman band of amorphous or disordered SiC.
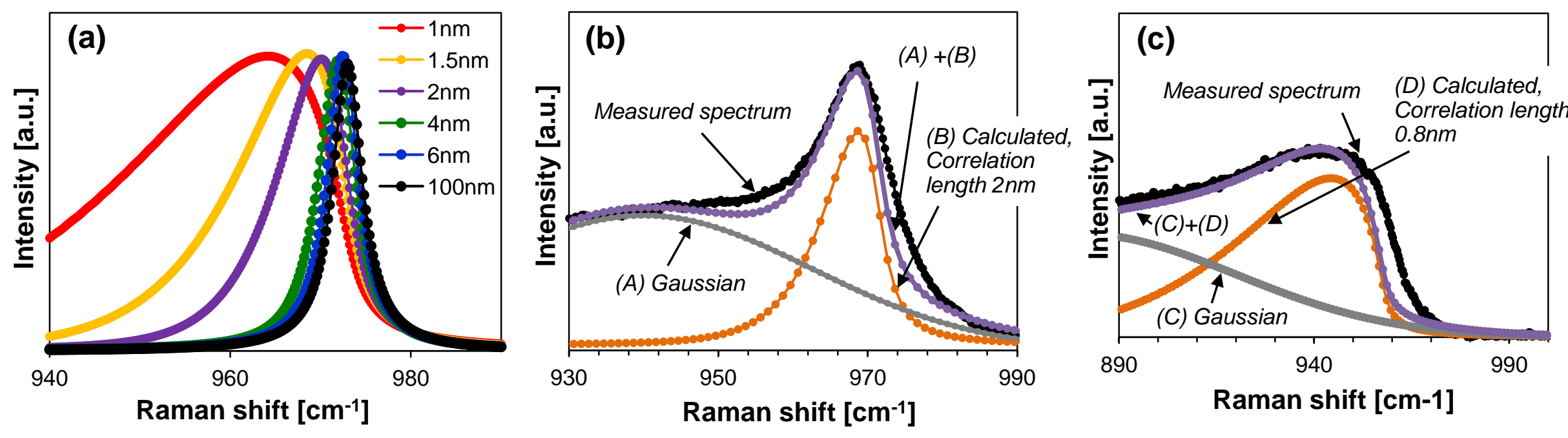
FIG. 4 Estimated defect distance calculated from Raman spectra plotted against irradiation temperature. Defect distances obtained by TEM, analyzing thermal conductivity, and simulation are also plotted. The number in red indicates neutron fluence for the Raman result (unit: dpa). The TEM micrograph inserted was taken by weak beam dark field imaging for $\mathrm{SiC}$ irradiated at $380^{\circ} \mathrm{C}$ to $0.11 \mathrm{dpa}$. Beam direction $(B)$ and $g$ vector are indicated near the image.

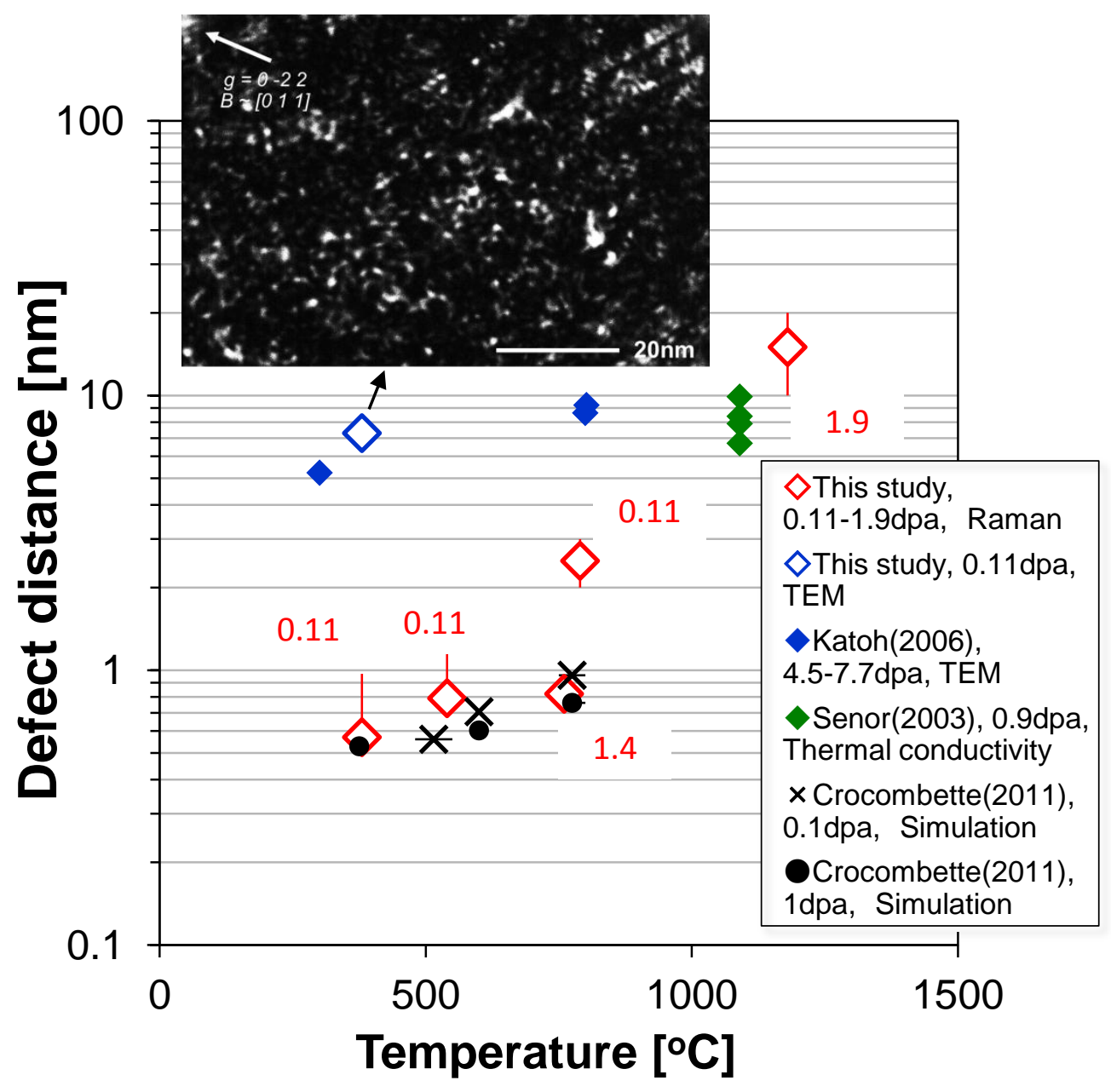


Graphical Abstract

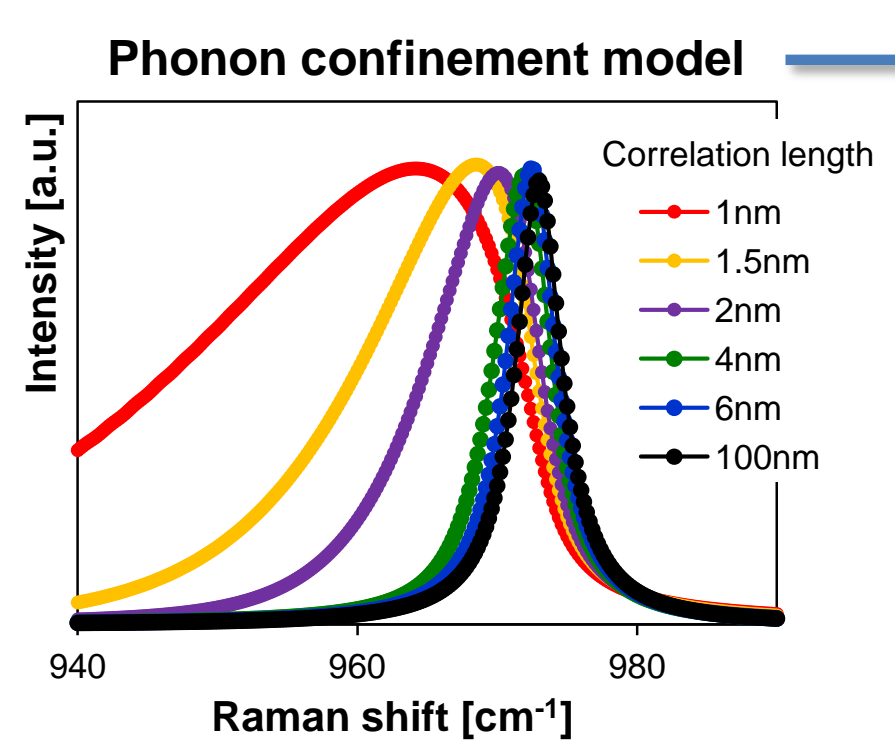

Measured and calculated Raman spectra of irradiated SiC

(B) Calculated,

Correlation length $2 \mathrm{~nm}$

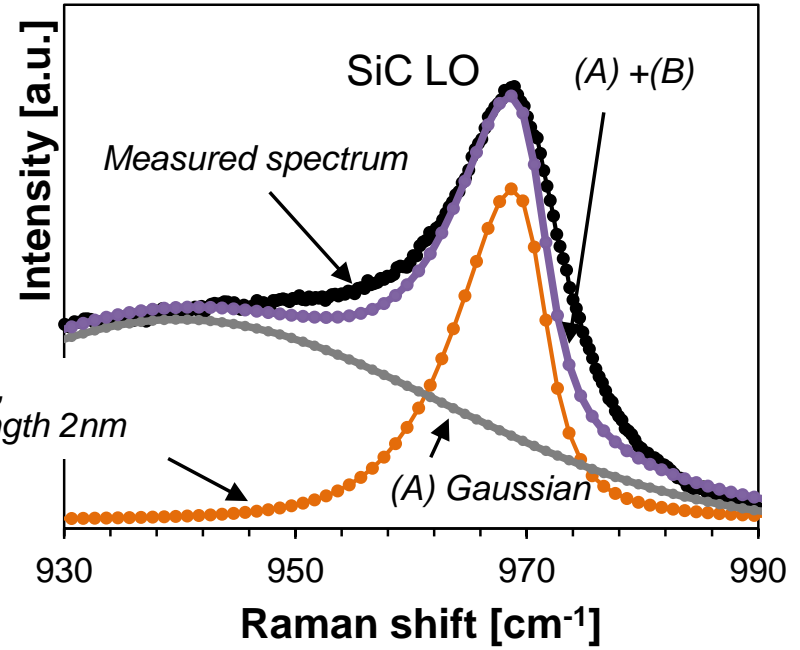

